\title{
Comparison and Extension of Free Dendritic Growth Models through Application to a Ag-15 Mass Pct Cu Alloy
}

\begin{abstract}
SELIS ÖNEL and TEIICHI ANDO
The formulations of existing free dendritic growth models were compared, and an extended model was proposed that employs a subregular solution model to compute the driving force for dendritic growth without Henrian restrictions. These models were also applied to a Ag-15 mass pct $\mathrm{Cu}$ alloy to numerically compare their predictions. Models that address only the thermal, solutal, and curvature supercoolings do not properly account for the interface kinetics, even with modifications with the kinetic partition coefficient and liquidus slope. It is only in models that account for the interfacial driving force, $-\Delta G^{*}$, that the kinetic supercooling is properly addressed. All of the models in comparison yield numerically similar predictions for the solutal growth regime, but models that employ the kinetic partition coefficient and liquidus slope, but do not address the interfacial driving force, fail to correctly describe the thermal control regime. The solutal-to-thermal transition is characterized by a rapid increase of interfacial driving force, which causes the tip temperature $T^{*}$ to increase with increasing growth rate $V$. The criterion for the transition stage is given as $d \ln \left(-\Delta G^{*}\right) / d \ln V>1$.
\end{abstract}

DOI: $10.1007 / \mathrm{s} 11661-008-9568-4$

(C) The Minerals, Metals \& Materials Society and ASM International 2008

\section{INTRODUCTION}

IN free dendritic growth, the latent heat of crystallization is dissipated mainly by the thermal diffusion into the supercooled liquid. Thus, the thermal gradient ahead of the growing dendrite is negative. Such a negative thermal gradient may be experienced by equiaxed dendritic grains growing in the interior of a solidifying ingot while competing with columnar dendritic grains growing from the mold wall. The interest in producing ingots with predominantly equiaxed grains initiated the study of free dendritic growth. Early free dendritic growth models, ${ }^{[1,2]}$ therefore, considered small melt supercoolings and local equilibrium conditions at the solid-liquid interface, as typically encountered in conventional casting.

In the early 1980s, the need for predicting the microstructural evolution in rapid solidification prompted the development of models that more properly described the free dendritic growth in supercooled melts. An early model of this kind was developed by Lipton, Glicksman, and Kurz (LGK). ${ }^{[3,4]}$ The LGK model incorporates the Ivantsov function ${ }^{[5]}$ to address the coupled heat and mass transport around a paraboloidal dendrite tip in steady-state free dendritic growth. ${ }^{[5]}$ Another important feature of the LGK model is the replacement of the extremum tip radius with one determined on the Kurz-Fisher stability criterion. ${ }^{[6]}$ The

TEIICHI ANDO, Professor, is with the Department of Mechanical and Industrial Engineering, Northeastern University, Boston, MA 02115. Contact e-mail: tando@coe.neu.edu SELIS ÖNEL, Full-Time Faculty Member, is with the Department of Chemical Engineering, Hacettepe University, Beytepe Campus, Ankara TR-06532, Turkey. Manuscript submitted December 2, 2007.

Article published online July 8, 2008 model also adopts the linear liquidus approximation from the earlier models, on which the total supercooling is defined with three components, namely, the thermal, solutal, and curvature supercoolings. The adoption of the three-component total supercooling necessarily fixes the interfacial liquid and solid compositions at the liquidus and the solidus, respectively. Such a local equilibrium interfacial condition, however, occurs only at low supercoolings.

To account for the nonequilibrium interfacial kinetics encountered in the free dendritic growth at higher supercoolings, Lipton, Kurz, and Trivedi (LKT) ${ }^{[7]}$ replaced the Kurz-Fisher stability criterion in the LGK model with the Trivedi-Kurz criterion, ${ }^{[8]}$ which applies to all values of Péclet numbers. However, the LKT model inherits the straight liquidus and solidus approximation and the three-component total supercooling, which limits its applicability to only low supercoolings. To address the nonequilibrium solute partitioning at high supercoolings, Trivedi, Lipton, and Kurz (TLK) ${ }^{[9]}$ extended the LKT model by incorporating the solute trapping equation presented by Aziz. ${ }^{[10]}$ The TLK model, as presented in Reference 9, however, still keeps the total supercooling consisting only of thermal, solutal, and curvature supercoolings, as well as straight phase boundaries.

Boettinger, Coriell, and Trivedi (BCT) were the first to correctly address the nonequilibrium solute partitioning at the solid-liquid interface in a free dendritic growth model. ${ }^{[11]}$ While the BCT model also uses the Aziz solute trapping model and the Trivedi-Kurz stability criterion, and is built on straight liquidus and solidus, it differs fundamentally from the previous two models because of its consistency with the thermodynamic requirement that the compositions at the solidliquid interface be in the range that gives a positive 
interfacial driving force, as addressed by Baker and Cahn. ${ }^{[12]}$ Also, the model satisfies the kinetic requirement that the interface migrate as the rate atoms attach themselves to the growing solid, which, at relatively low supercoolings, may be proportional to the interfacial driving force. ${ }^{[13]}$ The incorporation of the interfacial driving force allows the BCT model to define the total supercooling correctly with four components, namely, the thermal, solutal, curvature, and kinetic supercoolings. The BCT model can be extended for alloys of binary systems with curved phase boundaries, as shown by DiVenuti and Ando (DA). ${ }^{[14]}$

The LKT, TLK, and BCT models have been widely used because of their relatively simple analytic formulations, but not without limitations. The three-component total supercooling assumed in the LKT and TLK models in their original forms ${ }^{[7,9]}$ fixes the interfacial solute concentrations at local equilibrium values. The Aziz solute trapping model, used in the TLK and BCT models, has been instrumental but, as described in Reference 10, applies only to dilute alloys, although it can be readily replaced with an improved model. ${ }^{[5,16]}$ Henrian restrictions also apply to the Baker-Cahn equation $^{[12]}$ for the interfacial driving force employed in the BCT model. These limitations of the models have led some of their users to contrive modifications. The LKT and TLK models, originally defined only on the thermal, solutal, and curvature supercooling, have been used with the "kinetic liquidus slope",[11] and/or a "kinetic supercooling" in an effort to address the interface kinetics. ${ }^{[17-19]}$ The BCT model itself has been used with the kinetic liquidus slope in the Trivedi-Kurz criterion. ${ }^{[20-22]}$ The latter modification is also made in a recent BCT-based model ${ }^{[23]}$ in which the effects of nonFickian mass transport on solute partitioning are addressed. Such modifications, however, have not been validated.

One of the objectives of the present work was to elucidate the differences among the formulations of the existing free dendritic growth models (LKT, TLK, BCT, $\mathrm{DA}$, and modified models) and further provide a numerical comparison of these models through application to a Ag-15 mass pet $\mathrm{Cu}$ alloy. In addition, an extended model is proposed in which the interfacial driving force is directly computed with a thermodynamic solution model to lift the Henrian restriction on the Baker-Cahn equation. This modification, applied to the DA model in the present study, is also numerically tested by comparison with the existing models. The effects of the non-Fickian mass transport ${ }^{[23]}$ are not addressed, as they are not essential to the arguments presented in this article.

\section{COMPARISON OF EXISTING FREE DENDRITIC GROWTH MODELS}

All of the existing models described here (LKT, TLK, BCT, DA, and modified models) consider steady-state growth of a dendrite, represented by a paraboloid of revolution, into an alloy melt of composition $C_{0}$ supercooled to $T_{\infty}$ below the liquidus temperature $T_{L}$ $\left(C_{0}\right)$.

\section{A. LKT and TLK Models}

The original LKT model ${ }^{[7]}$ considers the total supercooling, defined by $\Delta T=T_{\infty}-T_{L}\left(C_{0}\right)$, to consist of three components, the thermal $\left(\Delta T_{t}\right)$, solutal $\left(\Delta T_{c}\right)$, and curvature $\left(\Delta T_{r}\right)$ supercoolings (Figure 1(a)). The three supercooling components are given, respectively, by the three terms on the right-hand side of

$$
\Delta T=\Delta T_{t}+\Delta T_{c}+\Delta T_{r}=\frac{\Delta H_{f}}{c_{p}} \mathrm{I}_{\mathrm{v}}\left(\mathrm{P}_{\mathrm{t}}\right)+m_{L}\left(C_{0}-C_{L}^{*}\right)+\frac{2 \Gamma}{r}
$$

where $\Delta H_{f}$ is the molar enthalpy of fusion, $c_{p}$ is the specific heat, $m_{L}$ is the slope of the equilibrium liquidus, $C_{L}^{*}$ is the solute concentration in the liquid at the interface, $\Gamma$ is the Gibbs-Thompson coefficient, $r$ is the tip radius, $I_{v}\left(P_{t}\right)$ is the Ivantsov function, ${ }^{[24,25]}$ and $P_{t}$ is the thermal Péclet number. Equation [1], the total supercooling equation, is solved for the tip velocity $V$ and the tip radius $r$ with the aid of the Trivedi-Kurz stability criterion, ${ }^{[8]}$ which, for free dendritic growth under the conditions of equal thermal diffusivity and conductivity, gives

$$
r=\frac{\Gamma / \sigma^{*}}{\frac{\mathrm{P}_{\mathrm{t}} \Delta H}{c_{p}} \xi_{t}-\frac{2 m_{L} \mathrm{P}_{\mathrm{c}} C_{0}\left(1-k_{0}\right)}{1-\left(1-k_{0}\right) \mathrm{I}_{\mathrm{v}}\left(\mathrm{P}_{c}\right)} \xi_{c}}
$$
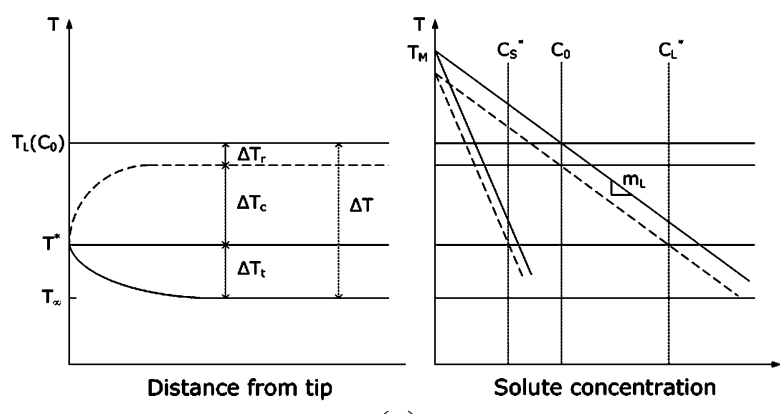

(a)
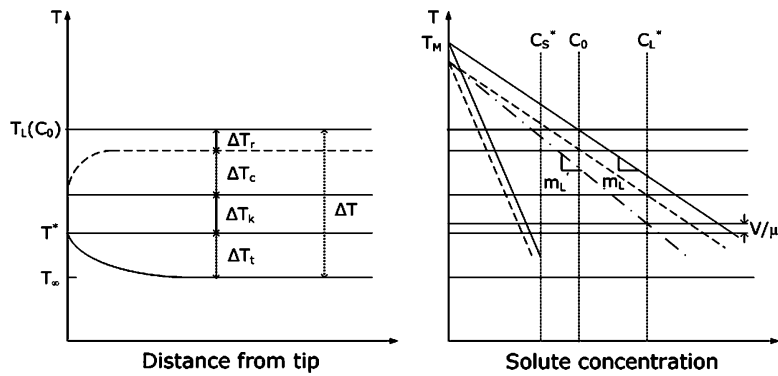

(b)

Fig. 1-Thermal and mass diffusion fields and supercooling components in free dendritic growth. (a) Local equilibrium at the tip. The total supercooling is comprised of three components, $\Delta T_{r}, \Delta T_{c}$, and $\Delta T_{t}$. (b) Nonequilibrium compositions at the tip. The total supercooling is comprised of four components, $\Delta T_{r}, \Delta T_{c}, \Delta T_{t}$, and $\Delta T_{k}$. 
where $\sigma^{*}$ is the stability parameter, ${ }^{[26-28]} \mathrm{P}_{\mathrm{c}}$ is the solutal Péclet number, $k_{0}$ is the equilibrium partition coefficient, and $\xi_{t}$ and $\xi_{c}$ are functions of, respectively, $\mathrm{P}_{t}$ and $\mathrm{P}_{\mathrm{c}}$ and $k_{0}$, as defined in Reference 8. Despite the adoption of the Trivedi-Kurz criterion, Eq. [2], which applies to all ranges of Péclet numbers, the LKT model, defined on the three supercooling components, only predicts local equilibrium interfacial solute concentrations. For cases where interface kinetic effects are important, $\mathrm{LKT}^{[7]}$ suggest to include in Eq. [1] a kinetic supercooling expressed as $V / \mu$, where $\mu$ is the interface kinetic coefficient. ${ }^{[8]}$ The validity of such a correction is discussed later in this section.

The TLK model, ${ }^{[9]}$ also built on the thermal, solutal, and curvature supercoolings, adopts the kinetic partition coefficient $k=C_{S}^{*} / C_{L}^{*}$ given by ${ }^{[10]}$

$$
k=\frac{k_{0}+\beta_{0} V}{1+\beta_{0} V}
$$

where $C_{S}^{*}$ is the solute concentration in the solid at the interface and $\beta_{0}$ is the solute trapping coefficient. The kinetic partition coefficient $k$ replaces the equilibrium partition coefficient $k_{0}$ in Eqs. [1] and [2]. Although these equations predict $C_{S}^{*} \approx C_{L}^{*} \approx C_{0}$ at high $V$, such predictions contradict the underlying assumption that the total supercooling consists only of the thermal $\left(\Delta T_{t}\right)$, solutal $\left(\Delta T_{c}\right)$, and curvature $\left(\Delta T_{r}\right)$ supercoolings, which necessarily requires that both $C_{S}^{*}$ and $C_{L}^{*}$ be fixed at the curvature-corrected solidus and liquidus at $T^{*}$, respectively (Figure 1(a)).

\section{B. BCT Model}

The latter dilemma is overcome in the BCT model ${ }^{[11]}$ by invoking the need to address the response function for the interface temperature and composition. ${ }^{[12]}$ For this purpose, BCT adopt a linear kinetic law: ${ }^{[13]}$

$$
V=-V_{0}\left(\frac{\Delta G^{*}}{\mathrm{R} T^{*}}\right)
$$

where $T^{*}$ is the interface temperature, $\Delta G^{*}$ is the free energy change across the interface, and $V_{0}$ is the maximum crystallization velocity. ${ }^{[13]}$ Equation [4] states that the interface velocity is singly dependent on the driving force, i.e., $-\Delta G^{*}$, which is a function of $T^{*}$ and $C_{L}^{*}$ (Figure 2). Equation [4] is derived from the more general form, $V=V_{0}\left[1-\exp \left(-\Delta G / \mathrm{R} T^{*}\right)\right]$, for the conditions where $-\Delta G / \mathrm{R} T^{*}$ is sufficiently small. ${ }^{[13]}$

Another key feature of this model is the adoption of the analytical expression for $\Delta G^{*}$ given by Baker and Cahn: ${ }^{[12]}$

$$
\frac{\Delta G^{*}}{\mathrm{R} T^{*}}=\left(1-C_{S}^{*}\right) \cdot \ln \frac{\left(1-C_{S}^{*}\right)\left(1-C_{L}^{e q}\right)}{\left(1-C_{L}^{*}\right)\left(1-C_{S}^{e q}\right)}+C_{S}^{*} \cdot \ln \frac{C_{S}^{*} C_{L}^{e q}}{C_{L}^{*} C_{S}^{e q}}
$$

where $C_{L}^{e q}$ and $C_{S}^{e q}$ are the equilibrium solute concentrations in mole fraction defined at $T^{*}$. Equation [5] is obtained on Henrian conditions and, as such, applies only to dilute alloys. ${ }^{[12]}$ Therefore, BCT, in combining Eqs. [4] and [5], apply further approximations by

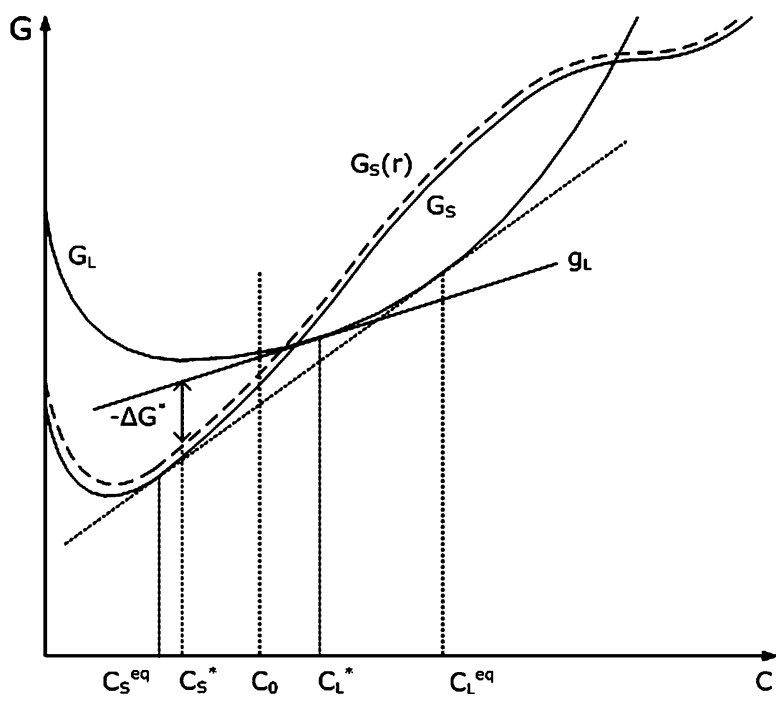

Fig. 2-Schematic illustrating the tangent-to-curve rule ${ }^{[12]}$ to determine the driving force, $-\Delta G^{*}$, for the growth of a dendrite with a tip radius $r$.

neglecting the products of mole fractions, which, after Gibbs-Thompson correction, yields

$$
T^{*}=T_{M}+m_{L}^{\prime} C_{L}^{*}-\frac{2 \Gamma}{r}-\frac{V}{\mu}
$$

where $T_{M}$ is the melting point of the pure metal, $\mu=V_{0}\left(k_{0}-1\right) / m_{L}$, and $m_{L}^{\prime}$ is the so-called kinetic liquidus slope, which is shown in Figure 1(b) and defined by ${ }^{[11]}$

$$
m_{L}^{\prime}=\frac{1-k\left(1-\ln \frac{k}{k_{0}}\right)}{1-k_{0}} m_{L}
$$

Equation [6] permits defining another supercooling component, $\Delta T_{k}$, the kinetic supercooling, which accounts for the nonequilibrium interfacial kinetics, as seen in Figure 1(b). While no ambiguity exists on the definitions of the thermal and curvature supercoolings, literature indicates that the division between the solutal and kinetic supercoolings has not always been clear to the users of the model. This ambiguity originates from Eq. [6], which, despite its correctness, may seem to suggest that the solutal supercooling be defined with the kinetic liquidus slope as $\Delta T_{c}=m_{L} C_{0}-m_{L}^{\prime} C_{L}^{*[22,29,30]}$ or $\Delta T_{c}=m_{L}^{\prime}\left(C_{0}-C_{L}^{*}\right){ }^{[23]}$ The correct expression, however, should be $\Delta T_{c}=m_{L}\left(C_{0}-C_{L}^{*}\right),{ }^{[4]}$ because the solutal supercooling by definition is the actual decrease of liquidus temperature caused by the solute buildup at the interface. With the latter definition of $\Delta T_{c}$, the kinetic supercooling should be what is left of the total supercooling, which, based on Eqs. [6] and [7], is given by ${ }^{[14]}$

$$
\begin{aligned}
\Delta T_{k} & =-\frac{m_{L} C_{0}\left[k_{0}-k\left(1-\ln \frac{k}{k_{0}}\right)\right]}{\left(1-k_{0}\right)\left[1-(1-k) \mathrm{I}_{\mathrm{v}}\left(\mathrm{P}_{\mathrm{c}}\right)\right]}+\frac{V}{\mu} \\
& =\left(m_{L}-m_{L}^{\prime}\right) C_{L}^{*}+\frac{V}{\mu}
\end{aligned}
$$


Thus, the $V / \mu$ term does not solely represent the kinetic supercooling except for pure metals, ${ }^{[8]}$ for which the first term in Eq. [8] is zero. For binary alloys, $\Delta T_{k}$ depends not only on $V$ but also on $C_{L}^{*}$ and varies from zero at low growth rates where $k=k_{0}$ and $V / \mu \approx 0$ to $m_{L} C_{0}\left(k_{0}-1-\ln k_{0}\right) /\left(k_{0}-1\right)+V / \mu$, or $\left[m_{L}-m_{L}^{\prime}(k=1)\right] C_{0}+V / \mu$, at very high growth rates where $m_{L}^{\prime}(k=1)$ is the slope of the $T_{0}$ line between the liquid and the solid, given by $m_{L}^{\prime}(k=1)=m_{L} \ln k_{0} /$ $\left(k_{0}-1\right) .{ }^{[11]}$ Thus, the first term in Eq. [8] can be regarded as the kinetic supercooling required to restrict solute partitioning at the interface.

Adding Eq. [8] to Eq. [1] gives the expression for the total supercooling of the BCT model:

$$
\begin{aligned}
\Delta T_{\mathrm{BCT}}= & \Delta T_{t}+\Delta T_{c}+\Delta T_{r}+\Delta T_{k} \\
= & \frac{\Delta H_{f}}{c_{p}} \mathrm{I}_{\mathrm{v}}\left(\mathrm{P}_{\mathrm{t}}\right)+m_{L} C_{0}\left[1-\frac{m_{L}^{\prime} / m_{L}}{1-(1-k) \mathrm{I}_{\mathrm{v}}\left(\mathrm{P}_{\mathrm{c}}\right)}\right] \\
& +\frac{2 \Gamma}{r}+\frac{V}{\mu}
\end{aligned}
$$

The BCT model combines Eq. [9] with the Aziz solute trapping kinetics, Eq. [3], and the Trivedi-Kurz criterion, Eq. [2], given on the kinetic partition coefficient $k$ instead of $k_{0}$, to solve them for $V, T^{*}, r, C_{L}^{*}$, and $k$.

\section{DA Model — an Extension of BCT Model}

The BCT model can be made applicable to curved liquidus and solidus that are often encountered in rapid solidification. For this purpose, DiVenuti and Ando $(\mathrm{DA})^{[14]}$ replace Eq. [6] with

$$
T^{*}=T_{L}\left(C_{L}^{*}\right)-\frac{2 \Gamma}{r}-\Delta T_{k}
$$

where $T_{L}(C)$ is the liquidus temperature as a function of solute concentration $C$. This permits writing Eq. [2] as ${ }^{[14]}$

$$
r=\frac{\Gamma / \sigma^{*}}{\frac{\mathbf{P}_{\mathrm{t}} \Delta H}{c_{p}} \xi_{t}-\frac{2\left(\frac{d T_{L}}{d C}\right)_{C=C_{L}^{*}} \mathrm{P}_{\mathrm{c}} C_{0}(1-k)}{1-(1-k) \mathbf{I}_{\mathrm{v}}\left(\mathbf{P}_{\mathrm{c}}\right)} \xi_{c}}
$$

which rigorously accounts for the curved liquidus in a manner consistent with the original Trivedi-Kurz criterion. ${ }^{[8]}$ We note that $d T_{L} / d C$ equals $m_{L}$, and not $m_{L}^{\prime}$, for a straight liquidus regardless of the value of $C_{L}^{*}$. Thus, the BCT model, as it is, correctly incorporates Eq. [11].

DA keep the Aziz solute trapping equation, Eq. [3], and, by eliminating the products of mole fractions, rewrite the linear kinetic law, Eq. [4], for curved liquidus and solidus as

$$
\begin{aligned}
& C_{S}^{e q}\left(T^{*}+\Delta T_{r}\right)-C_{L}^{e q}\left(T^{*}+\Delta T_{r}\right)+\frac{2 \mathrm{P}_{\mathrm{c}} D}{V_{0} R} \\
& +\frac{C_{o}\left\{1-k\left[1-\ln k+\ln C_{S}^{e q}\left(T^{*}+\Delta T_{r}\right)-\ln C_{L}^{e q}\left(T^{*}+\Delta T_{r}\right)\right]\right\}}{1+(1-k) \mathrm{I}_{\mathrm{v}}\left(\mathrm{P}_{\mathrm{c}}\right)} \\
& \quad=0
\end{aligned}
$$

Note that Eq. [12] is equivalent to Eq. [6] if $T_{L}(C)=T_{M}+m_{L} C$. Thus, the DA model reduces to the BCT model for linear liquidus and solidus.

\section{Modified Models}

The success of the BCT model has led some users of previous models to use them with modifications based on Eq. [6] in an effort to more properly address the nonequilibrium interface kinetics at high Péclet numbers. These modifications are characterized by the introduction of a "kinetic supercooling" $V / \mu$ or the replacement of the equilibrium liquidus slope $m_{L}$ with the kinetic liquidus slope $m_{L}^{\prime}$ defined by Eq. [7]..$^{[7,17-19]}$ For example, a TLK-based model with such modifications is described on

$$
\begin{gathered}
\Delta T_{\text {Mod-TLK }}=\frac{\Delta H_{f}}{c_{p}} \mathrm{I}_{\mathrm{v}}\left(\mathrm{P}_{\mathrm{t}}\right)+m_{L}^{\prime}\left(C_{0}-C_{L}^{*}\right)+\frac{2 \Gamma}{r}+\frac{V}{\mu} \\
r=\frac{\Gamma / \sigma^{*}}{\frac{\mathrm{P}_{\mathrm{t}} \Delta H}{c_{p}} \xi_{t}-\frac{2 m_{L}^{\prime} \mathrm{P}_{\mathrm{c}} C_{0}\left(1-k_{0}\right)}{1-\left(1-k_{0}\right) \mathbf{I}_{\mathrm{v}}\left(\mathbf{P}_{\mathrm{c}}\right)} \xi_{c}}
\end{gathered}
$$

both of which clearly differ from those of the BCT model. Thus, such modifications fail to remedy the previous models. This is also understood from the fact that Eqs. [6] and [7], as we have seen earlier, come from the linear kinetic law, Eq. [4], and the Baker-Cahn equation, Eq. [5], neither of which applies to the previous models. The modified Trivedi-Kurz criterion with $m_{L}^{\prime}$, Eq. [14], has been used also with the BCT model, ${ }^{[20-23]}$ but such a modification is an unnecessary correction of the already correct BCT model. ${ }^{[14,29-31]}$

\section{EXTENTION FOR NONDILUTE ALLOYS}

While the existing models have been useful and widely used in rapid solidification studies, they are still limited to dilute alloys due to the Henrian restrictions placed on the Baker-Cahn and Aziz equations. In the present work, a new approach to calculating the interfacial driving force with a temperature-dependent subregular solution model ${ }^{[32]}$ is introduced to circumvent at least the restriction on the Baker-Cahn equation. The solution model devised after Gaskell ${ }^{[33]}$ and Murray, ${ }^{[34]}$ but with a modification to make the model more self-consistent, is represented by

$$
\begin{aligned}
& G=C_{1} G_{1}^{\mathrm{o}}+C_{2} G_{2}^{\mathrm{o}}+\mathrm{R} T\left(C_{1} \ln C_{1}+C_{2} \ln C_{2}\right)+G^{X S} \\
& G^{X S}=C_{1} \mu_{1}^{X S}+C_{2} \mu_{2}^{X S}=\left(A+B C_{2}\right) C_{1} C_{2}\left(1-\frac{T}{\tau}\right)
\end{aligned}
$$




$$
\mu_{1}^{X S}=G^{X S}+C_{2} \frac{\partial G^{X S}}{\partial C_{1}}=\left[A+B\left(2 C_{2}-1\right)\right]\left(1-\frac{T}{\tau}\right) C_{2}^{2}
$$

$$
\mu_{2}^{X S}=G^{X S}+C_{1} \frac{\partial G^{X S}}{\partial C_{2}}=\left(A+2 B C_{2}\right)\left(1-\frac{T}{\tau}\right) C_{1}^{2}
$$

where $G$ and $G^{X S}$ are, respectively, the Gibbs free energy and excess free energy of binary solutions of components 1 and 2; $C_{i}$ and $\mu_{i}^{X S}$ are, respectively, the mole fraction and the excess chemical potential of component $i$; and $A, B$, and $\tau$ are constants, which need to be determined for the phases of interest. The terms $G, G^{X S}$, and $\mu_{i}^{X S}$ in these expressions are fully consistent with each other, i.e., $G^{X S}=C_{1} \mu_{1}^{X S}+C_{2} \mu_{2}^{X S}$.

Equations [15] through [18] permit calculating the interfacial driving force $-\Delta G^{*}$ by the tangent-to-curve rule $^{[12]}$ illustrated in Figure 2; i.e.,

$$
\Delta G^{*}=G_{S}\left(T^{*}, C_{S}^{*}\right)+\frac{2 \Gamma \Delta S}{r}-g_{L}\left(T^{*}, C_{S}^{*}\right)
$$

where $G_{S}\left(T^{*}, C_{S}^{*}\right)$ is the free energy of the solid at $C_{S}^{*}, \Delta S$ is the molar entropy of fusion, and $g_{L}$ is the equation of the line tangent to the $G_{L}$ curve at $C_{L}^{*}$. Substituting Eq. [19] in Eq. [4] then yields an equation that replaces Eq. [6] of the BCT model or Eq. [12] of the DA model. We keep the Aziz equation, Eq. [3], in this model despite its Henrian limitations, but it can be readily replaced with an improved model such as the ones presented by Aziz and Kaplan ${ }^{[15]}$ and Abbaschian and Kurz. ${ }^{[16]}$

\section{NUMERICAL COMPARISON OF THE MODELS}

The existing models (Section II) and the extended DA model (Section III) were numerically compared by applying them to the free dendritic growth in a Ag-23 at. pct $\mathrm{Cu}(\mathrm{Ag}-15$ mass pet $\mathrm{Cu})$ alloy. The choice of this alloy was justified on the basis of the availability of thermodynamic data ${ }^{[35-37]}$ and the opportunity for verification with the prior computation results presented in Reference 11.

The value of $m_{L}$ required in the LKT, TLK, and BCT models, and also in the modified TLK model defined by Eqs. [3], [13], and [14], was determined on the $\mathrm{Ag}-\mathrm{Cu}$ phase diagram to be -520.6 in $\mathrm{K} /$ mole fraction using the straight line between the melting point of $\mathrm{Ag}$ and the point on the liquidus at $C_{0}(15$ mass pct or 0.23 in mole fraction). The mole fraction-based value of $k_{0}$ was then determined to be 0.393 from the $C_{S}^{e q} / C_{L}^{e q}$ at the eutectic temperature. The curved equilibrium liquidus and solidus and their metastable extensions required in the DA and extended DA models were calculated with the subregular solution model using the data in the literature. $^{[35-37]}$ Figure 3 shows the calculated equilibrium and metastable phase equilibria. The values of the other parameters used in the present study are shown in Table I.

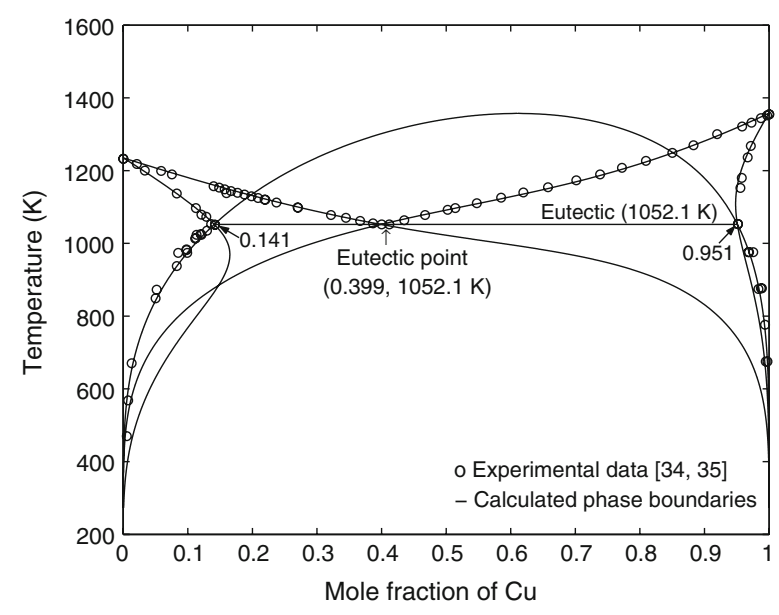

Fig. 3-Ag-Cu phase diagrams calculated with the subregular solution model. ${ }^{[32]}$ Metastable extensions of liquidus and solidus and metastable miscibility gap are also shown.

Table I. Parameters for Ag-23 At. Pct Cu Alloy Used in the Present Work

\begin{tabular}{ll}
\hline$T_{M}$ & $1234 \mathrm{~K}$ \\
$m_{L}$ & $-520.594 \mathrm{~K} / \mathrm{mol}$ fraction \\
$k_{0}$ & 0.393 mole fraction $/$ mol fraction \\
$\Delta H_{f} / c_{P}$ & $374 \mathrm{~K}$ \\
$D$ & $2.1 \times 10^{-9} \mathrm{~m}^{2} / \mathrm{s}$ for TLK, modified TLK, \\
& $\quad$ and $\mathrm{BCT}$ models \\
$D\left(T^{*}\right)$ & $=D_{0} e^{-E /\left(\mathrm{R} T^{*}\right)}$ for DA and extended \\
& $\quad \mathrm{DA}$ models $\left(D_{0}=1 \times 10^{-7} \mathrm{~m}^{2} / \mathrm{s}\right.$, \\
& $\left.\quad E=36 \times 10^{3} \mathrm{~J} / \mathrm{mol}\right)$ \\
$\alpha$ & $\quad 6.6 \times 10^{-5} \mathrm{~m}^{2} / \mathrm{s}$ \\
$1 / \beta_{0}$ & $2 \mathrm{~m} / \mathrm{s}$ for TLK, modified TLK, and BCT models \\
& $D\left(T^{*}\right) / a_{0}$ for DA and extended DA models \\
& $\left(a_{0}=5 \times 10^{-10} \mathrm{~m}\right)$ \\
$V_{0}$ & $2000 \mathrm{~m} / \mathrm{s}$ \\
$\Gamma$ & $1.53 \times 10^{-7} \mathrm{mK}$ \\
\hline
\end{tabular}

Figure 4 compares the values of $V, r, k, C_{S}^{*}$, and $C_{L}^{*}$ calculated with the six models. At supercoolings up to about $85 \mathrm{~K}$, all the models predict similar behaviors of $V, r, k, C_{S}^{*}$, and $C_{L}^{*}$. This is expected at low to moderate supercoolings where the kinetic supercooling is insignificant and solute diffusion-controlled growth prevails. At higher supercoolings, all the models, except the LKT model, predict a sharp jump of $V, r$, and $k$ to higher values (Figures 4(a) through (c)). The corresponding values of $C_{S}^{*}$ and $C_{L}^{*}$ also change abruptly, with both converging to $C_{0}$ (Figure $4(\mathrm{~d})$ ).

The abrupt changes in $V, r, k, C_{S}^{*}$, and $C_{L}^{*}$ reflect the transition from solute diffusion-controlled growth to thermal diffusion-controlled growth, ${ }^{[9,11]}$ which is verified in Figure 4(c) where the value of $k$ increases sharply from that of $k_{0}$ to nearly unity. The BCT, DA, and extended DA models predict similar transition behaviors over a relatively narrow range of $\Delta T$ beginning approximately at $85 \mathrm{~K}$. The TLK and modified TLK models, however, yield nonunique values of $V, r, k, C_{S}^{*}$, and $C_{L}^{*}$ over a $\Delta T$ range from about 50 or $65 \mathrm{~K}$ to $85 \mathrm{~K}$. Previous studies also presented such nonunique values of $V^{[9,20]}$ The LKT model, which assumes local 


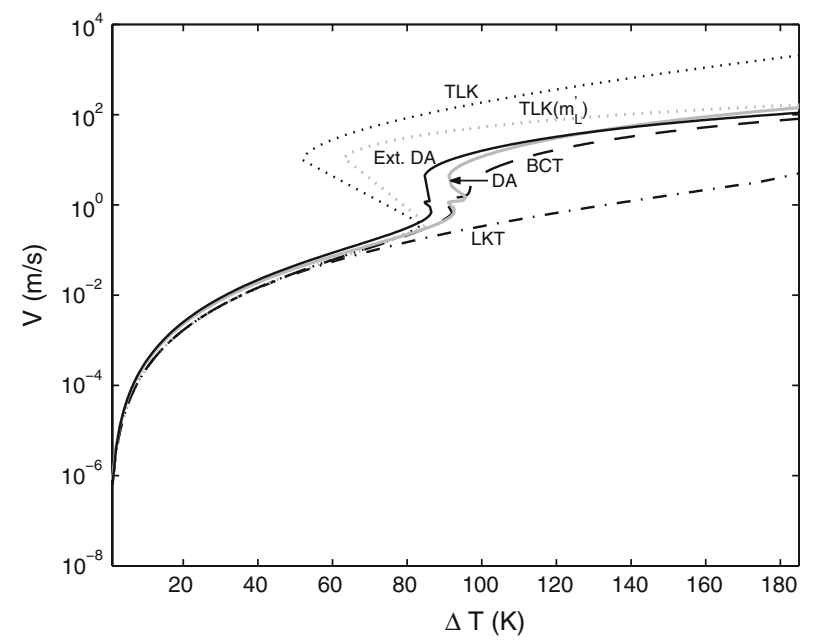

(a)

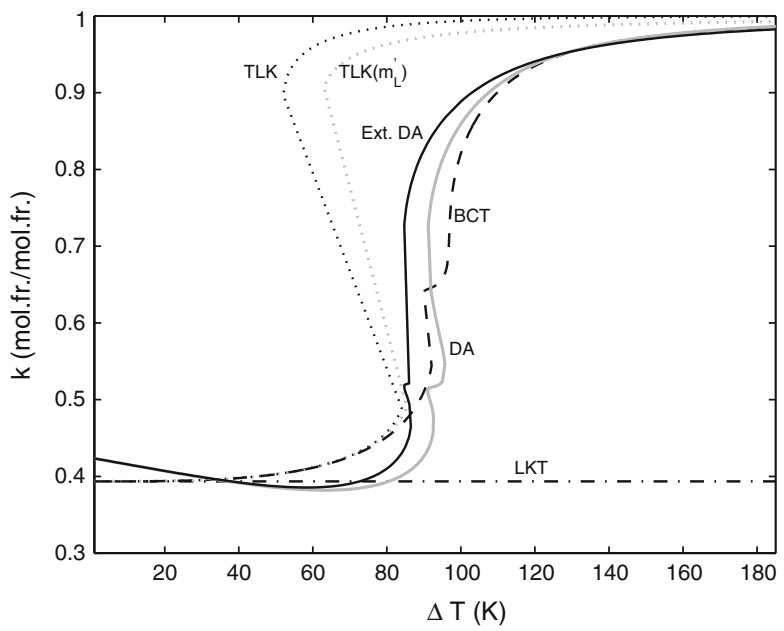

(c)

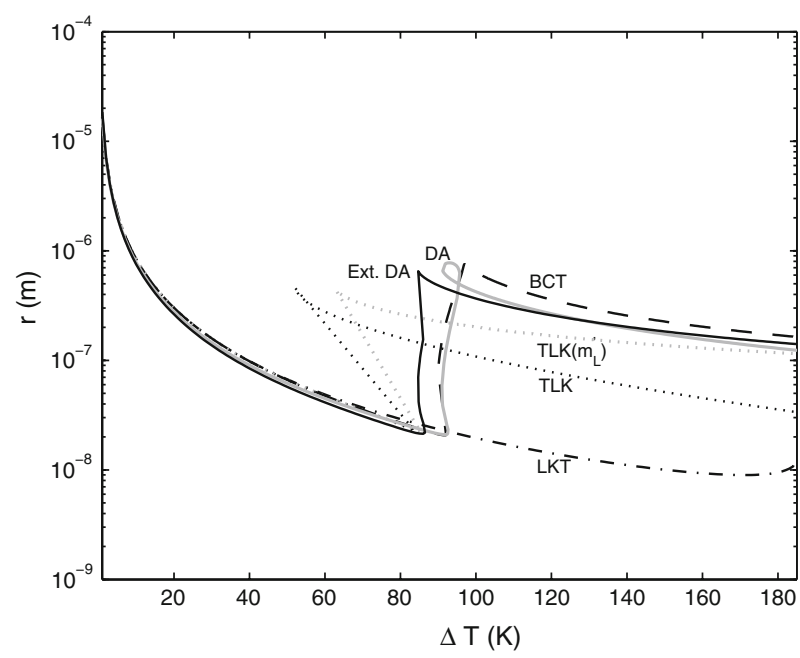

(b)

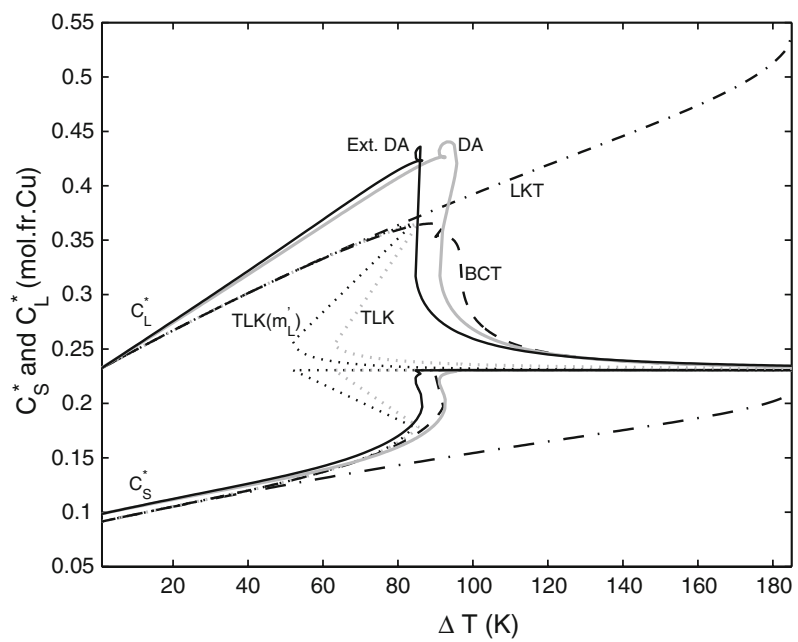

(d)

Fig. 4-Predicted kinetics of free dendritic growth in Ag-15 mass pct $\mathrm{Cu}$ as a function of total supercooling: (a) tip velocity, (b) tip radius, $(c)$ partition coefficient, and $(d)$ solute concentrations at tip.

equilibrium and no interface kinetic effects, predicts no transition to thermal growth.

While the transition from solute to thermal diffusion control is a well-understood phenomenon, ${ }^{[11]}$ whether these models correctly predict the transition still needs to be verified. For this purpose, we examine if the predicted values of $C_{S}^{*}$ and $C_{L}^{*}$ would actually give the interface a positive driving force for migration at the predicted value of $T^{*}$.

Figure 5 shows $T^{*}$ vs $\Delta T$ predicted by the models in comparison. Examine first the predictions by the TLK model. At $\Delta T=60 \mathrm{~K}$, two values of $T^{*}$ are predicted, i.e., $T^{*}$ may be 1054 or $1108 \mathrm{~K}$. Suppose $T^{*}=1054 \mathrm{~K}$, then the predicted value of partition coefficient $k$ is 0.411 (Figure 4(c)), which is only slightly higher than $k_{0}$. Thus, solute diffusion-controlled growth would be predicted if $T^{*}$ were $1054 \mathrm{~K}$. For this condition, $C_{S}^{*}$ and $C_{L}^{*}$ are predicted to be 0.137 and 0.333 , respectively (Figure 4(d)). The curvature-corrected values of $C_{S}^{e q}$

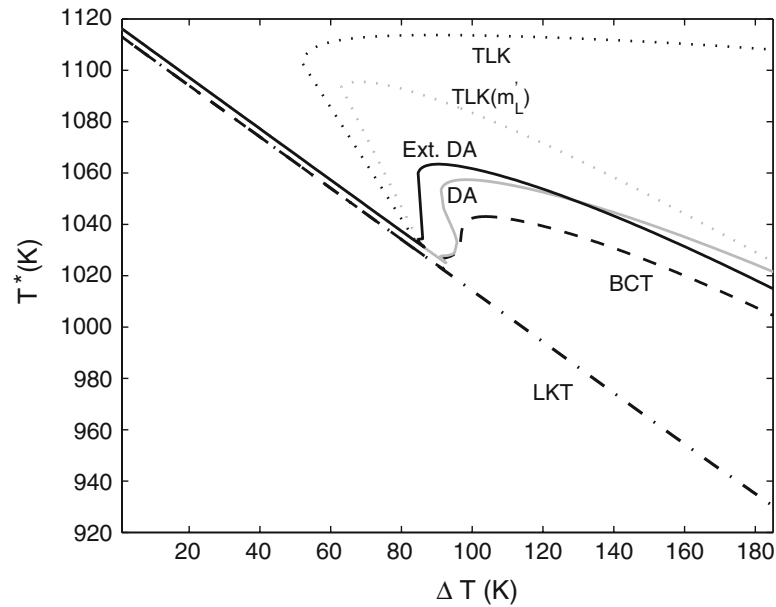

Fig. 5-Predicted variations of tip temperature with total supercooling. 
and $C_{L}^{e q}$ at $1054 \mathrm{~K}$ are calculated from $T^{*}=T_{M}+m_{L} C-2 \Gamma / r$ with the predicted value of tip radius $\left(r=4.73 \times 10^{-8} \mathrm{~m}\right)$ and the equilibrium partition coefficient $\left(k_{0}=0.393\right)$ to be 0.131 and 0.333 , respectively. Thus, the interface remains essentially at local equilibrium, as expected from the three-component supercooling adopted in this model. When substituted in Eq. [5], these values of $C_{S}^{*}, C_{L}^{*}, C_{S}^{e q}$, and $C_{L}^{e q}$ at $1054 \mathrm{~K}$ give $-\Delta G^{*}=0.23 \mathrm{~J} / \mathrm{mol}$, which gives $V \approx 0.06 \mathrm{~m} / \mathrm{s}$ when substituted in Eq. [4], while the TLK model itself predicts a similar value of $V$ for the diffusion-controlled growth at $\Delta T=60 \mathrm{~K}$ (Figure 4(a)). Thus, for diffusioncontrolled growth, the TLK model yields predictions that are numerically consistent with those by the models that account for the interfacial driving force.

If $T^{*}$ were $1108 \mathrm{~K}$ at $\Delta T=60 \mathrm{~K}, k$ would be 0.96 (Figure 4(c)). Thus, thermal diffusion-controlled growth is predicted. The terms $C_{S}^{*}$ and $C_{L}^{*}$ are, respectively, 0.231 and 0.240 (Figure 4(d)). With the predicted value of tip radius $\left(r=2.78 \times 10^{-7} \mathrm{~m}\right)$, the curvature-corrected $C_{S}^{e q}$ and $C_{L}^{e q}$ at $1108 \mathrm{~K}$ are calculated to be 0.094 and 0.240 . Thus, $C_{L}^{*}=C_{L}^{e q}$ (local equilibrium with respect to $C_{L}^{*}$ ) is predicted, which contradicts the prediction of thermal growth. This dilemma obviously results from the assumed three-component supercooling, which necessarily fixes $C_{L}^{*}$ at $C_{L}^{e q}$. The value of $C_{S}^{*}(0.231)$, which is much larger than $C_{S}^{e q}(0.094)$, is predicted only because the predicted high growth rate (Figure 4(a)) gives a high value of $k(0.96)$ through Eq. [3]. With these values of concentrations, Eq. [5] yields $-\Delta G^{*}=-741 \mathrm{~J} / \mathrm{mol}$, suggesting that no growth by thermal control would actually be possible under these conditions. Figure 6 schematically illustrates how such a negative driving force would result at $1108 \mathrm{~K}$.

At supercoolings above about $85 \mathrm{~K}$, the TLK model predicts only thermal diffusion-controlled growth (Figure 4). Although the occurrence of growth by thermal diffusion control itself is not argued, whether it occurs in the manner predicted should still be examined. As a test, we estimate $-\Delta G^{*}$ at $\Delta T=100 \mathrm{~K}$, where $T^{*}=1110 \mathrm{~K}$ (Figure 5); $k=0.994$ (Figure 4(c)); $C_{S}^{*}$ and $C_{L}^{*}$ are, respectively, 0.231 and 0.232 (Figure 5(d)); and $C_{S}^{e q}$ and $C_{L}^{e q}$ at $1110 \mathrm{~K}$ corrected for the predicted value of tip radius $\left(r=1.08 \times 10^{-7} \mathrm{~m}\right)$ are, respectively, 0.916 and 0.233 . The same procedure with Eq. [5] yields $-\Delta G^{*}=-785 \mathrm{~J} / \mathrm{mol}$. Thus, thermal diffusioncontrolled dendrite growth is not possible in the manner predicted. In fact, the predicted values of $T^{*}$ in Figure 5 are much higher than the $T_{0}$ temperature of the alloy (estimated, for the straight liquidus and solidus, to be $\left.1050 \mathrm{~K}^{[11]}\right)$; i.e., thermal diffusion-controlled growth would not be possible at such a high $T^{*}$.

The failure of the TLK model in properly predicting the thermal control regime is caused by the inappropriate adoption of the solute trapping equation (Eq. [3]) in a model that is based only on $\Delta T_{t}, \Delta T_{c}$, and $\Delta T_{r}$, i.e. (Eq. [1]). In such a model, $C_{L}^{*}$ is necessarily fixed at the curvature-corrected local equilibrium value at $T^{*}$. Consequently, the condition $k \approx 1$, required for thermal diffusion-controlled growth, is satisfied only if $T^{*} \approx$ $T_{L}\left(C_{0}\right)$. For such a condition, $C_{S}^{*} \approx C_{L}^{*} \approx C_{L}^{e q}=$ $C_{S}^{e q} / k_{0} \approx C_{0}=0.23$ and Eq. [5] reduces to

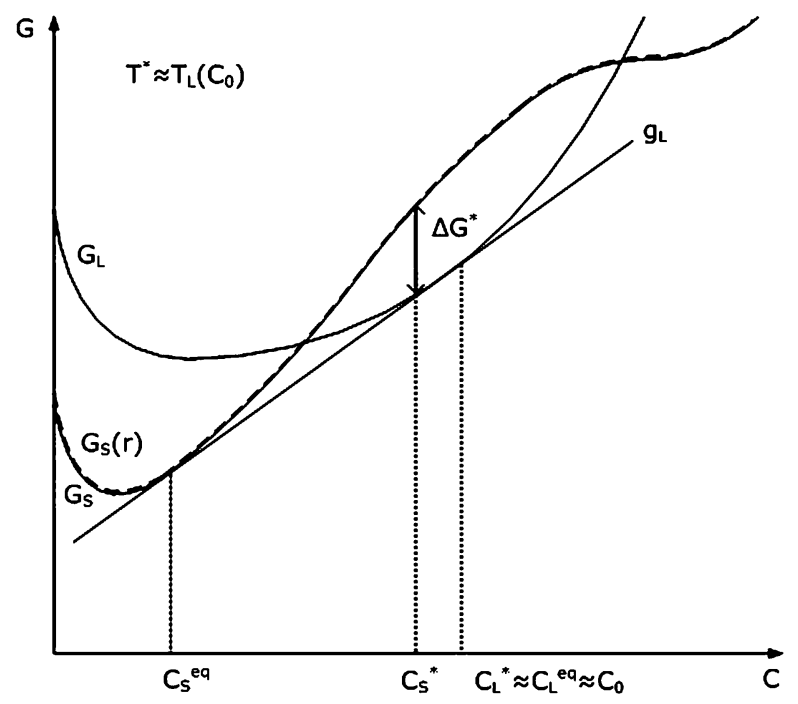

Fig. 6-Thermal growth at a tip temperature near the liquidus temperature is not possible, since the interfacial driving force $\left(-\Delta G^{*}\right)$ is negative.

$$
\frac{\Delta G^{*}}{\mathrm{R} T^{*}} \approx\left(1-C_{0}\right) \cdot \ln \frac{(1-C)}{\left(1-k_{0} C_{0}\right)}-C_{0} \cdot \ln k_{0}
$$

which yields $-\Delta G^{*} \approx-803 \mathrm{~J} / \mathrm{mol}$; i.e., no thermal growth is possible.

Figure 5 also suggests that the modified TLK model, despite the adoption of the $V / \mu$ term in Eq. [13] and the kinetic liquidus slope $m_{L}^{\prime}$ in Eqs. [13] and [14], also fails to properly address the thermal diffusion regime as the predicted values of $T^{*}$ exceed $T_{0}(1050 \mathrm{~K})$ at $\Delta T<155 \mathrm{~K}$. In addition, Eq. [5] yields a negative driving force. For example, the driving force at $\Delta T=100 \mathrm{~K}$, where $T^{*}=1080 \mathrm{~K}, k=0.980, C_{S}^{*}=$ $0.231, C_{L}^{*}=0.236, r=2.02 \times 10^{-7} \mathrm{~m}, C_{S}^{\mathrm{eq}}=0.115$, and $C_{L}^{\mathrm{eq}}=0.293$, is calculated to be $-382 \mathrm{~J} / \mathrm{mol}$. At higher supercoolings, where $T^{*}<T_{0}, \Delta T_{\text {Mod-TLK }}$, given by Eq. [13], becomes similar to $\Delta T_{\mathrm{BCT}}$, given by Eq. [9], and the predictions by the modified TLK model approach those by the BCT model, as seen in Figures 4 and 5. Thus, the modified TLK model numerically agrees with the BCT model at very high supercoolings.

The BCT model predicts the $T^{*}$ values to be sufficiently below $T_{0}$ (1050 K for straight liquidus and solidus) at all values of $\Delta T$ in the thermal diffusion regime (Figure 5). The DA and extended DA models also satisfy the condition $T^{*}<T_{0}$ for thermal diffusion-controlled growth. $\left(T_{0}=1072 \mathrm{~K}\right.$ if computed with the subregular solution model.) Also, the BCT, DA, and extended DA models all predict a positive interfacial driving force for all values of $\Delta T$ (Figure 7). The predicted values of driving force exhibit full parallelism to those of growth rate shown in Figure 4(a), reflecting the adoption of the linear kinetic law of Eq. [4] in these models. The values of the driving force predicted by the three models are smaller, by an order of magnitude, than those estimated from the $C_{L}^{*}$ and $C_{S}^{*}$ values predicted by the TLK and modified TLK models, thus yielding lower and more reasonable values of $V$. 
The values of $T^{*}$ predicted by the TLK, modified TLK, BCT, DA, and extended DA models decrease with increasing $V$ until the solutal-to-thermal transition sets in, where $T^{*}$ increases to a maximum as full transition to thermal control is achieved at a sufficiently high value of $V$ (Figure 8). Such a $T^{*}-V$ inversion has been predicted to occur during the transition from solutal dendritic growth to planar thermal growth under constrained growth conditions ${ }^{[38-40]}$ and also during the solutal-to-thermal transition in free dendritic growth. ${ }^{[17,20]}$ It should be noticed, however, that full transition to thermal growth must occur such that the maximum value of $T^{*}$ still stays below $T_{0}$. The latter

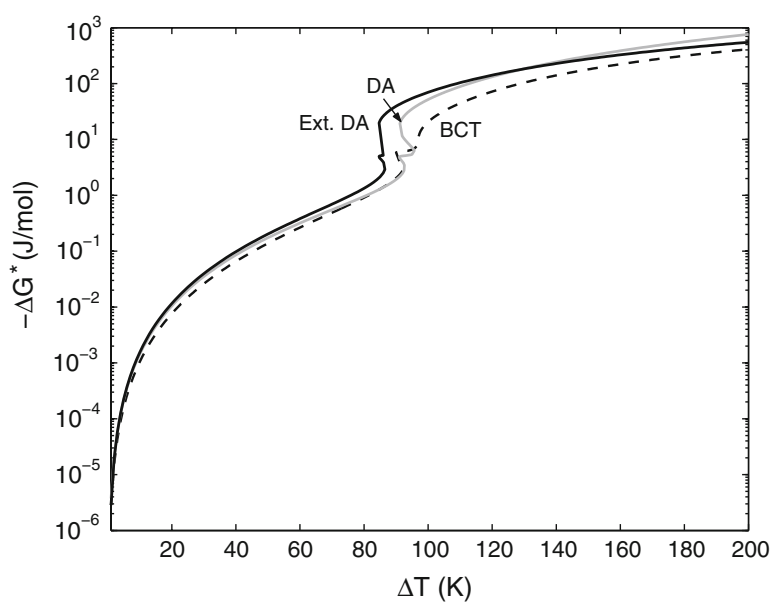

Fig. 7-Predicted interfacial driving force $\left(-\Delta G^{*}\right)$ vs total supercooling. Positive values of driving force are assured by the use of Eq. [15] in the BCT model and Eq. [19] in the DA and extended DA models.

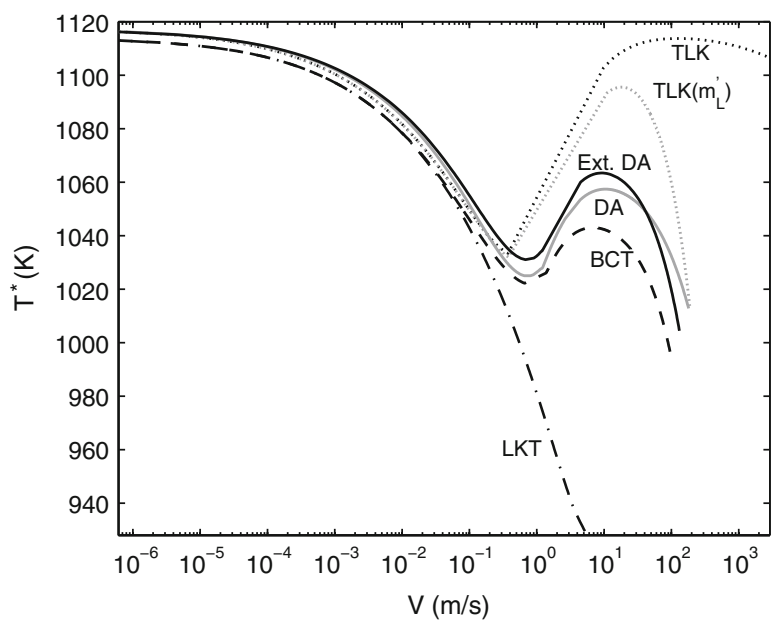

Fig. 8- $T^{*}$ vs $V$ predicted by different models. All models predict numerically similar values of $T^{*}$ at low values of $V$, where the growth rate is limited by solute diffusion in the liquid. The $T^{*}-V$ inversion reflects the transition from solutal-to-thermal control. For the linear kinetic law, as assumed in the BCT, DA, and extended DA models, the criterion of the transition stage is given as $d \ln \left(-\Delta G^{*}\right) / d \ln V>1$. condition is satisfied in the BCT, DA, and extended DA models.

The origin of the $T^{*}-V$ inversion may be understood by rewriting Eq. [4] as

$$
T^{*}=\frac{V_{0}\left(-\Delta G^{*}\right)}{R} \cdot \frac{1}{V}
$$

At low supercoolings where solutal growth prevails, the increase in driving force is still insignificant (Figure 7) and Eq. [21] predicts a nearly hyperbolic decrease of $T^{*}$ with increasing $V$. At higher supercoolings, increased solute trapping causes the values of $C_{L}^{*}$ and $C_{S}^{*}$ to deviate from the local equilibrium values (Figures 4(d) and 9) and the driving force begins to build up. Equation [21] suggests that a $T^{*}-V$ inversion, i.e., $d T^{*} / d V>0$, would result if the driving force increases more rapidly than $V$, or more specifically if $d\left(-\Delta G^{*}\right) / d V>\mathrm{R} T^{*} / V_{0}$, or $d \ln \left(-\Delta G^{*}\right) / d \ln V>1$. Thus, the latter condition defines the solutal-to-thermal transition stage. The calculated free energy curves in Figure 10 show how the shift of $C_{L}^{*}$ increases $g_{L}\left(T^{*}, C_{S}^{*}\right)$, and hence $-\Delta G^{*}$, from a low value at the beginning of the transition to a high value toward the end of the transition. At yet higher supercoolings, the growth rate increases rapidly as partitionless (massive) growth sets in, hence causing $T^{*}$ to decrease again. Thus, the $T^{*}-V$ inversion, i.e., the solute-to-thermal transition, can be understood properly only in a model that addresses the interfacial driving force.

\section{CONCLUSIONS}

The differences in the formulations of existing free dendritic growth models were discussed and an extended model was proposed that employs a subregular solution model to compute the driving force for dendritic growth. These models were applied to a Ag-15 mass pct $\mathrm{Cu}$ alloy to numerically compare their predictions. The following are the major conclusions reached.

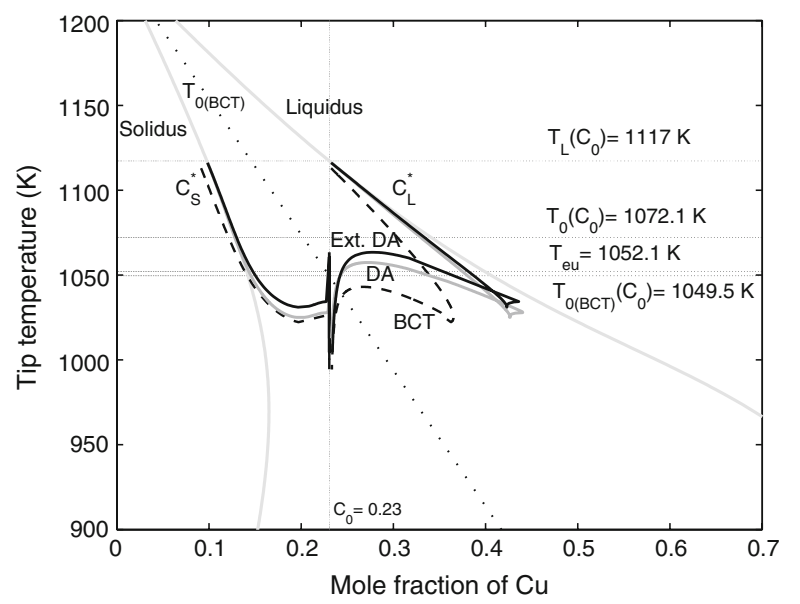

Fig. 9-Interfacial solute concentrations calculated as a function of tip temperature. 


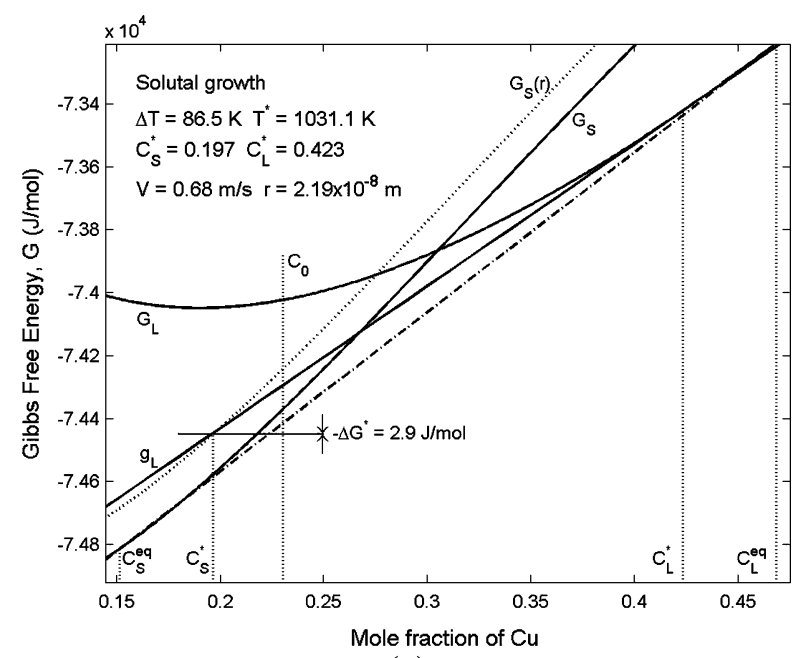

(a)

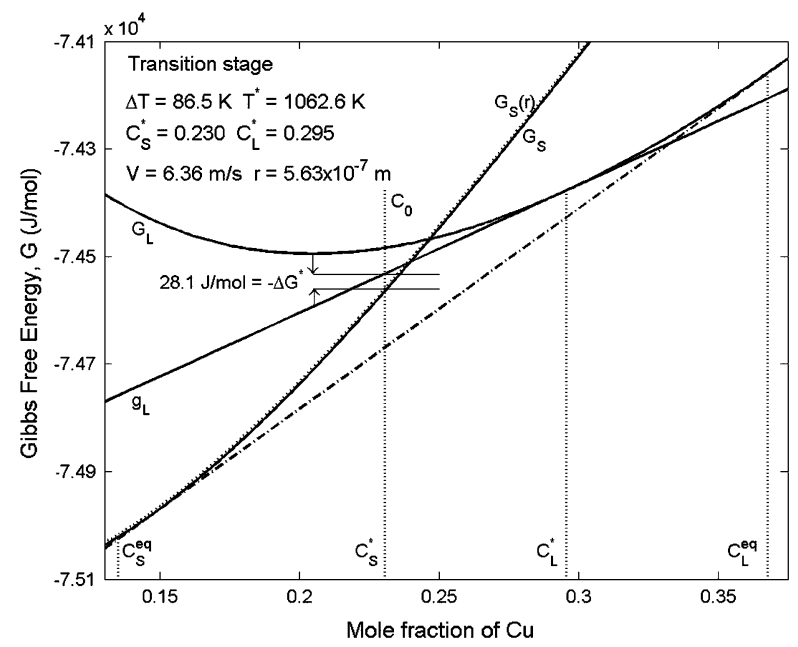

(b)

Fig. 10-Increase in driving force from (a) a low value typical of solutal growth at the beginning of the transition stage to $(b)$ a high value near the end of the transition where thermal growth sets in.

1. Models that are based only on the thermal, solutal, and curvature supercoolings (e.g., LKT and TLK) do not properly account for the interface kinetics, even with the inclusion of $V / \mu$ in the total supercooling and the replacement of the equilibrium partition coefficient and liquidus slope, $k_{0}$ and $m_{L}$, with the kinetic partition coefficient and liquidus slope, $k$ and $m_{L}^{\prime}$, defined, respectively, by Eqs. [3] and [7].

2. The BCT model and its extensions (DA and extended DA) correctly address the interface kinetics by adopting a linear kinetic law. The kinetic supercooling, as well as the thermal, solutal, and curvature supercoolings, is properly defined in these models. The kinetic supercooling, defined by $\Delta T_{k}=\Delta T-\Delta T_{t}-\Delta T_{c}-\Delta T_{r}$, has a component that depends on $C_{L}^{*}$ in addition to the $V / \mu$ term, as seen explicitly in Eq. [8] of the BCT model. The $\Delta T_{k}$ reduces to $V / \mu$ only for pure metals.

3. Equations [6] and [7] for the interface (tip) temperature $T^{*}$ and the kinetic liquidus slope $m_{L}^{\prime}$ given in the BCT model are obtained by combining the linear kinetic law and the Baker-Cahn equation for the interfacial driving force, and as such do not apply to models that do not properly address the interfacial driving force.

4. The Baker-Cahn equation for the interfacial driving force used in the BCT and DA models can be replaced with a thermodynamic solution model to circumvent the Henrian restrictions.

5. All the models in comparison yield numerically similar predictions for the solutal growth regime. Models that employ $T^{*}, m_{L}^{\prime}$, and $k$ but do not address the interfacial driving force can still predict the occurrence of the transition from the solutal-tothermal control. However, the values of $T^{*}$ predicted for the thermal regime far exceed $T_{0}$, and those of $C_{L}^{*}$ and $C_{S}^{*}$ yield a negative driving force. Models that account for the interfacial driving force (BCT and its extensions) properly predict the thermal growth regime.

6. The solutal-to-thermal transition stage is characterized by a rapid buildup of interfacial driving force, which causes $T^{*}$ to increase with $V$. Based on the linear kinetic law, the criterion for the transition stage is given as $d \ln \left(-\Delta G^{*}\right) / d \ln V>1$.

\section{REFERENCES}

1. D.E. Temkin: Sov. Phys.-Crystallogr., 1965, vol. 7, pp. 354-57.

2. R. Trivedi and W.A. Tiller: Acta Metall., 1978, vol. 25, pp. 679-87.

3. J. Lipton, M.E. Glicksman, and W. Kurz: Mater. Sci. Eng., 1984, vol. 65 , pp. 57-63.

4. J. Lipton, M.E. Glicksman, and W. Kurz: Metall. Trans. A, 1987, vol. 18 , pp. $341-45$

5. G.P. Ivantsov: Dokl. Akad. Nauk, USSR, 1947, vol. 58, pp. 56-57.

6. W. Kurz and D.J. Fisher: Acta Metall., 1981, vol. 29, pp. 11-20.

7. J. Lipton, W. Kurz, and R. Trivedi: Acta Metall., 1987, vol. 35 (4), pp. 957-64.

8. R. Trivedi and W. Kurz: Acta Metall., 1987, vol. 34 (8), pp. $1663-$ 70.

9. R. Trivedi, J. Lipton, and W. Kurz: Acta Metall., 1987, vol. 35 (4), pp. 965-70.

10. M.J. Aziz: J. Appl. Phys, 1982, vol. 53 (2), pp. 1158-68.

11. W.J. Boettinger, S.R. Coriell, and R. Trivedi: in Rapid Solidification Processing: Principles and Technologies IV, R. Mehrabian and P.A. Parish, eds., Claitor's Publishing Division, Baton Rouge, LA, 1988 , pp. $13-25$.

12. C. Baker and J.W. Cahn: Solidification, ASM, Metals Park, OH, 1971, pp. 23-58.

13. D. Turnbull: J. Phys. Chem., 1962, vol. 66, pp. 609-13.

14. A.G. DiVenuti and T. Ando: Metall. Mater. Trans. A, 1998, vol. 29 A, pp. $3047-56$.

15. M.J. Aziz and T. Kaplan: Acta Metall., 1988, vol. 36, pp. 2335-44.

16. R. Abbaschian and W. Kurz: in Solidification Processes and Microstructures: a Symp. in Honor of Wilffred Kurz, M. Rappaz, C. Beckermann, and R. Trivedi, eds., TMS, Warrendale, PA, 2004, pp. 314-24.

17. G.-X. Wang, V. Prasad, and S. Sampath: Metall. Mater. Trans. A, 2000, vol. 31A, pp. 735-46.

18. Y. Sasajima and M. Ichimura: J. Jpn. Inst. Light Met., 2003, vol. 53 (2), pp. 67-73.

19. D.M. Matson, J. Kertz Yurko, and R.W. Hyers: in Solidification Processes and Microstructures - a Symp. in Honor of Rohit Trivedi, M. Rappaz, C. Beckermann, and R. Trivedi, TMS, Warrendale, PA, 2004, pp. 205-10.

20. S.-G. Kim, S.-H. Shin, T. Suzuki, and T. Umeda: Metall. Mater. Trans. A, 1994, vol. 25A, pp. 2815-26. 
21. M. Suzuki, T.J. Piccone, M.C. Flemings, and H.D. Brody: Metall. Trans. A, 1991, vol. 22A, pp. 2761-68.

22. X.R. Liu, C.D. Cao, and B. Wei: Scripta Mater., 2002, vol. 46, pp. $13-18$.

23. P.K. Galenko and D.A. Danilov: J. Cryst. Growth, 1999, vol. 197, pp. 992-1002.

24. G.P. Ivantsov: Growth of Crystals, Consultants Bureau, New York, NY, 1958, vol. 1, pp. 76-85.

25. G.P. Ivantsov: in Growth of Crystals, Consultants Bureau, New York, NY, 1962, vol. 3, pp. 53-60.

26. S. Langer and H. Müller-Krumbhaar: Acta Metall., 1978, vol. 26, pp. 1681-87.

27. J.S. Langer and H. Müller-Krumbhaar: Acta Metall., 1978, vol. 26, pp. 1689-95.

28. J.S. Langer and H. Müller-Krumbhaar: Acta Metall., 1978, vol. 26, pp. 1697-08.

29. W. Loser and D.M. Herlach: Metall. Trans. A, 1992, vol. 23A, pp. 1585-91.

30. K. Eckler, R.F. Cochrane, D.M. Herlach, B. Feuerbacher, and M. Jurisch: Phys. Rev. B, 1992, vol. 45 (9), pp. 5019-22.
31. T. Suzuki and K. Sakuma: JSIJ Int., 1995, vol. 35 (2), pp. 178-82. 32. S. Önel: Ph.D. Thesis, Northeastern University, Boston, MA, 2006.

33. D.R. Gaskell: Introduction to the Thermodynamics of Materials, 4th ed., Taylor \& Francis, New York, NY, 2003, p. 252.

34. J.L. Murray: Metall. Trans. A, 1984, vol. 15A, pp. 261-68.

35. P. Subramanian and J.H. Perepezko: J. Phase Equilibria, 1993, vol. 14 (1), pp. $62-75$

36. C.T. Heycock and F.H. Neville: Phil. Trans. R. Soc. (London) A, 1897 , vol. 189 , pp. $25-69$

37. M. Binnewies and E.M. Milke: Thermochemical Data of Elements and Compounds, Wiley-VCH, New York, NY, 1999, pp. 12 and 434.

38. A. Karma and A. Sarkissian: Phys. Rev. Let., 1962, vol. 68, pp. 2616-19.

39. M. Carrard, M. Germaud, M. Zimmermann, and W. Kurz: Acta Metall. Mater., 1992, vol. 40, pp. 983-96.

40. W. Boettinger and M.J. Aziz: Acta Metall., 1989, vol. 37 (12), pp. 3379-91. 